\title{
Security Associations Management (SAM) Model for IP Multimedia System (IMS)
}

\author{
Muhammad Sher, Thomas Magedanz \\ Technical University Berlin \& Fokus Fraunhofer Institute, \\ Kaiserin-Augusta-Allee 31, D-10589 Berlin, Germany \\ \{sher, magedanz\}@fokus.fraunhofer.de \\ http://www.av.cs.tu-berlin.de/ \\ http://www.fokus.fraunhofer.de/ngni
}

\begin{abstract}
In this paper we propose Security Associations Management (SAM) model which consists of seven security associations \& managements based on different technical specifications of Third Generation Partnership Project (3GPP) [1] to develop Secure Service Provisioning Framework (SSPF) [2] for IP Multimedia System (IMS) at IMS Playground within Third Generation beyond (3Gb) Testbed [3] at Fokus, Fraunhofer. The objective of this enhanced security management model is to combine all security associations into single article that deal with the mutual authentication of user and network; to provide security across different interfaces like Ut interface (for HTTP services), Gm interface (air contact) between IMS client and IMS Core, Cx and Dx interfaces (between Home Subscriber Server HSS and IMS core network). It also deals with security when the user is roaming or in home network and security considerations for access networks. The main emphasis of SAM is to propose complete security protection model for IMS network and to the user, therefore only brief description of each security association is provided to understand the architecture and conceptual security model.
\end{abstract}

Key Words: Security Associations, Security Management, Key Management, Encryption, Network Domain Security, IP Multimedia Systems

\section{Introduction}

Security and information protection are the focal and central points for all data networks and telecommunication systems but with the emerging of fixed and mobile networks convergence like VoIP, IPv6, WLAN, IP Multimedia System (IMS), Universal Mobile Telecommunication Systems (UMTS) and General Packet Radio System (GPRS) etc., network security becomes critical and important to protect the networks as well as to manage secure communication between users.

Please use the following format when citing this chapter:

Sher, M. and Magedanz, 'T., 2007, in IlIP International lederation for Information Processing, Volume 229, Network Control and Engineering for QoS, Security, and Mobility, IV, ed. Gaïti, D., (Boston: Springer), pp. 311-325. 
As we know that all IP based networks are open and distributed nature of architecture which can enable easy access to services, information, and resources, together with the constant abuse of hackers, curious individuals, fraudsters, and organized crime units. Therefore complex security techniques and mechanisms such as secure data transmission, confidentiality, authentication, data integrity, anti-replay protection and intrusion detection system are the important security consideration features for all IP networks and mobile telecommunication systems.

The proposed security management model is based on different technical specifications of Third Generation Partnership Project (3GPP) [4-7] and consists on seven security associations to provide Secure Service Provisioning Framework (SSPF) [2] for IP Multimedia System (IMS) Playground within 3Gb Testbed at Fokus Fraunhofer [3]. In section 2 we describe briefly IMS technology and Fokus IMS Playground. In Section 3 we present the architecture of proposed security management model along with the security associations and the last section concludes the paper.

\section{IMS Technology and IMS Testbed}

In the prospect of global trends, the mobile communications world has defined within the evolution of cellular systems an all-IP network vision which integrates cellular networks and the Internet. This is the IP Multimedia System (IMS), namely overlay architecture for the provision of multimedia services, such as VoIP and videoconferencing on top of globally emerging $3 \mathrm{G}$ broadband packet networks. The IMS has been standardized by 3GPP [1] and 3GPP2 [8] in the beginning of this decade and is planned for deployment in 3G wireless networks around 2005/2006. Due to the fact that the IMS overlay architecture is widely abstracted from the air interfaces, the IMS can be used for any mobile access network technology as well as for fixed line access technology as currently promoted by ETSI TISPAN within the NGN reference architecture definition [9]. It is important to note, that the IMS defines service provision architecture, and as such can be seen as the next generation service delivery platform framework.

The important entities of IMS are given as:

- P-CSCF (Proxy Call State/Session Control Function):- It behaves like a proxy accepting requests and services.

- I-CSCF (Interrogating Call State Control Function):- It assigns S-CSCF to a user performing SIP registration, charging and resource utilisation.

- S-CSCF (Serving Call State Control Function):- It performs the session control services for the endpoint.

- MRF (Media Resource Function):- It provides media stream processing like media mixing, media announcements, media analysis and media transcoding.

- HSS (Home Subscription Function):- It is the master database of an IMS that stores IMS user profiles.

- AS (Application Server):- It provides service platform in IMS environment. 


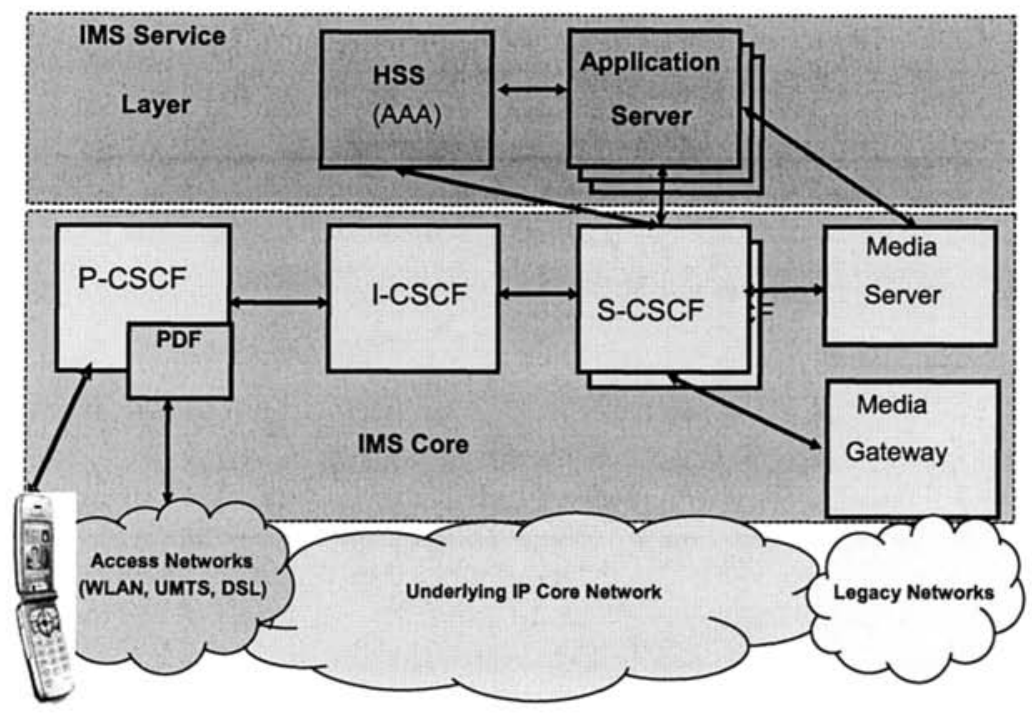

Fig. 1. IMS Architecture

In face of this and knowing the current challenges within the telecommunications market are mainly a consequence of insufficient early access to new enabling technologies by all market players, the Fraunhofer Institute FOKUS, known as a leading research institute in the field of open communication systems, has established with support from the German ministry of Education and Research (BMBF) a 3G beyond Testbed, known as "National Host for 3Gb Applications" [3]. This Testbed provides technologies and related know-how in the field of fixed and wireless next generation network technologies and related service delivery platforms.

As such a Testbed is quite complex by its very nature, FOKUS has coined in addition the notion of technology focused "playgrounds". One of these is the "Open IMS Playground @ FOKUS" [3] where you can find different access technologies, infrastructure components and management tools. FOKUS implemented all core components of the IMS and enriched this base infrastructure with components from commercial vendors. Particularly the field of SDP diversity, promoted within the IMS, is supported by providing different service platforms, such as Open Service Access (OSA) /Parlay, JAIN Service Logic Execution Environments (SLEE), Web Services / Parlay X, SIP Servlets, Call Processing Language (CPL), etc. on top of a multiplicity of access technologies including an exclusive 3G UMTS cell [10]. 


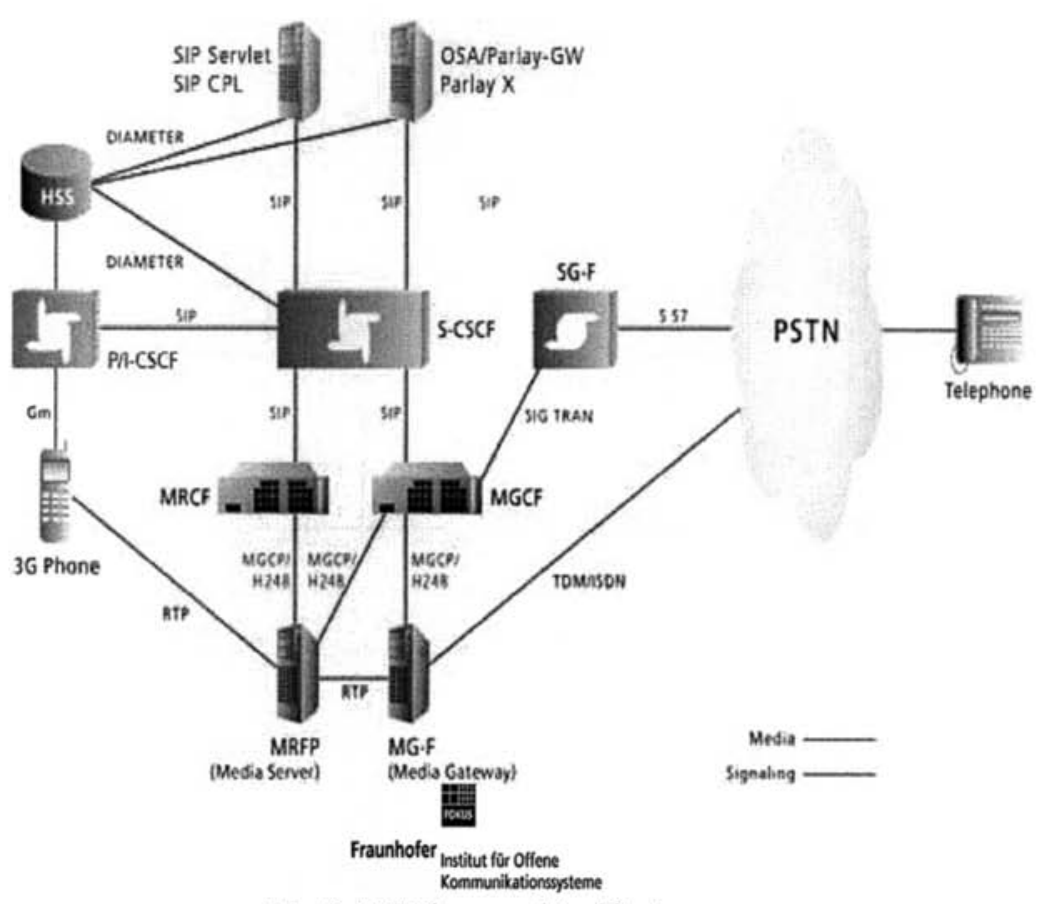

Fig. 2. IMS Playground Architecture

\section{IMS Security Management Model}

The overall security for IP Multimedia System (IMS) as standardized by 3GPP [1] in its different releases is summarized in diagram 3 and involves following security associations agreements and recommendations:

- Authentication \& Key Agreement between IM subscriber and home network

- Security Mechanism Agreement between IM client and visited network

- Integrity Protection and Confidentiality

- Network Domain Security between different Domains

- Existing GPRS/UMTS Access Security 


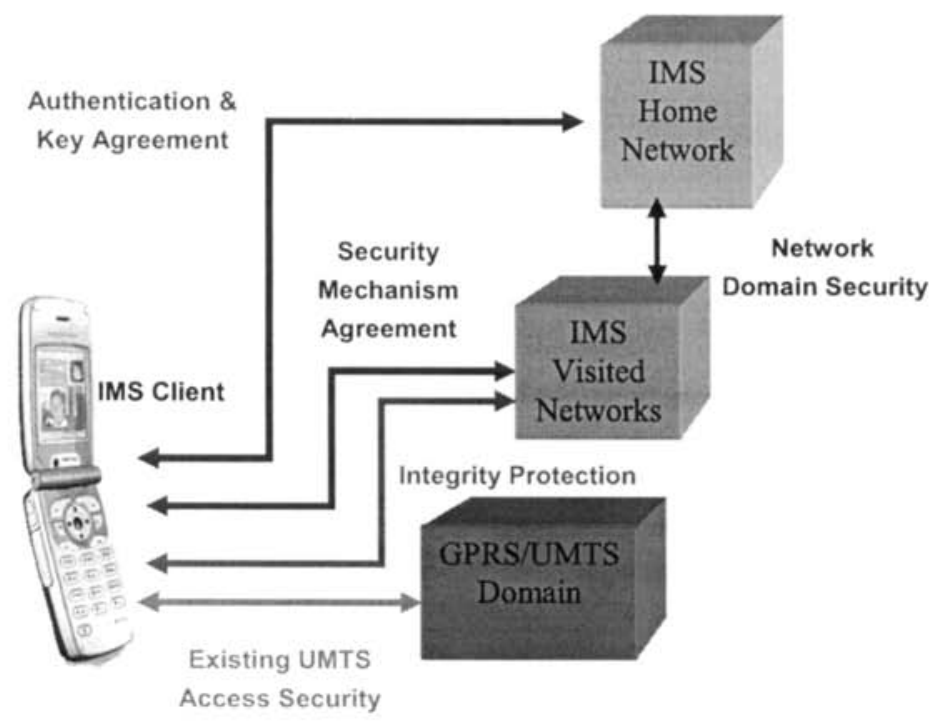

Fig. 3. Overall IMS Security

The architecture of proposed security management model of Secure Service Provisioning Framework (SSPF) for IP Multimedia System (IMS) is provided in figure 4, which presents the following seven security associations (SA1 to SA7) that are mandatory to protect IMS environment for secure and safe communication over wireless and wireline networks in circuit switched (CS) domain as well as packet switched (PS) domain.

- SA1: Authentication and Key Agreement between User and Network

- SA2: Security Management to Protect Gm-interface (Air Interface)

- SA3: Security Management for Cx and Dx interfaces (between IMS core \& HSS)

- SA4: Security Management for Roaming Users (visited network)

- SA5: Security Management for Home User (home network)

- SA6: Security Management for HTTP Services (Ut interface)

- SA7: Security Management for Access Networks (GPRS, UMTS, WLAN etc.)

Now we discuss briefly each Security Association and Agreement one by one. 


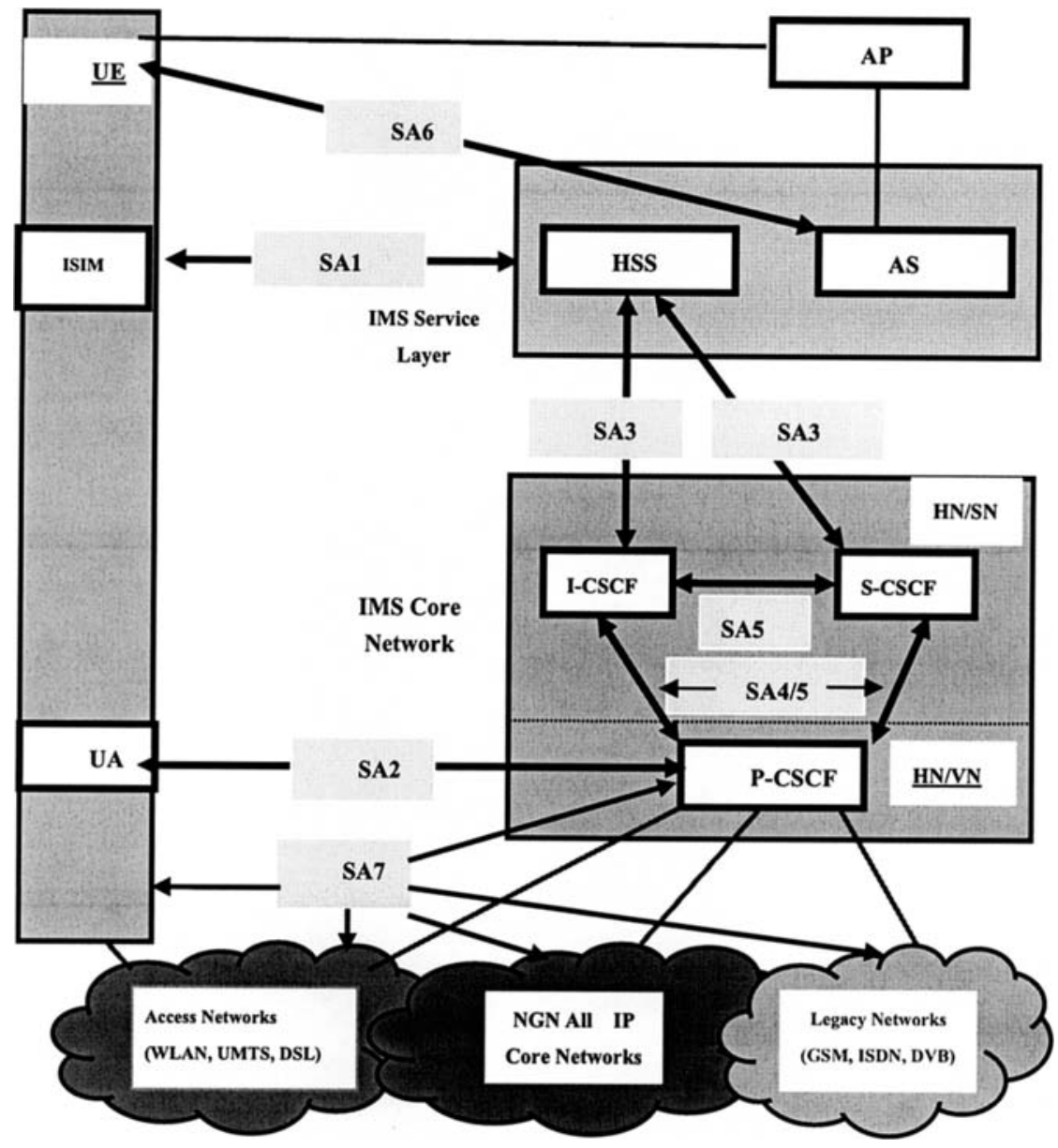

Fig. 4. IMS Security Management Model

\subsection{SA1: AKA Management between User and Network}

It provides mutual authentication of user and network. The Home Subscriber Server (HSS) is responsible for generating keys and challenges and after that it delegates the performance of subscriber authentication to the Serving-Call State Control Function (S-CSCF). The subscriber will have one user private identity which is called IP Multimedia Private Identity (IMPI) for authentication purpose and one external user public identity which is called IP Multimedia Public Identity (IMPU) which is used for routing purpose. 


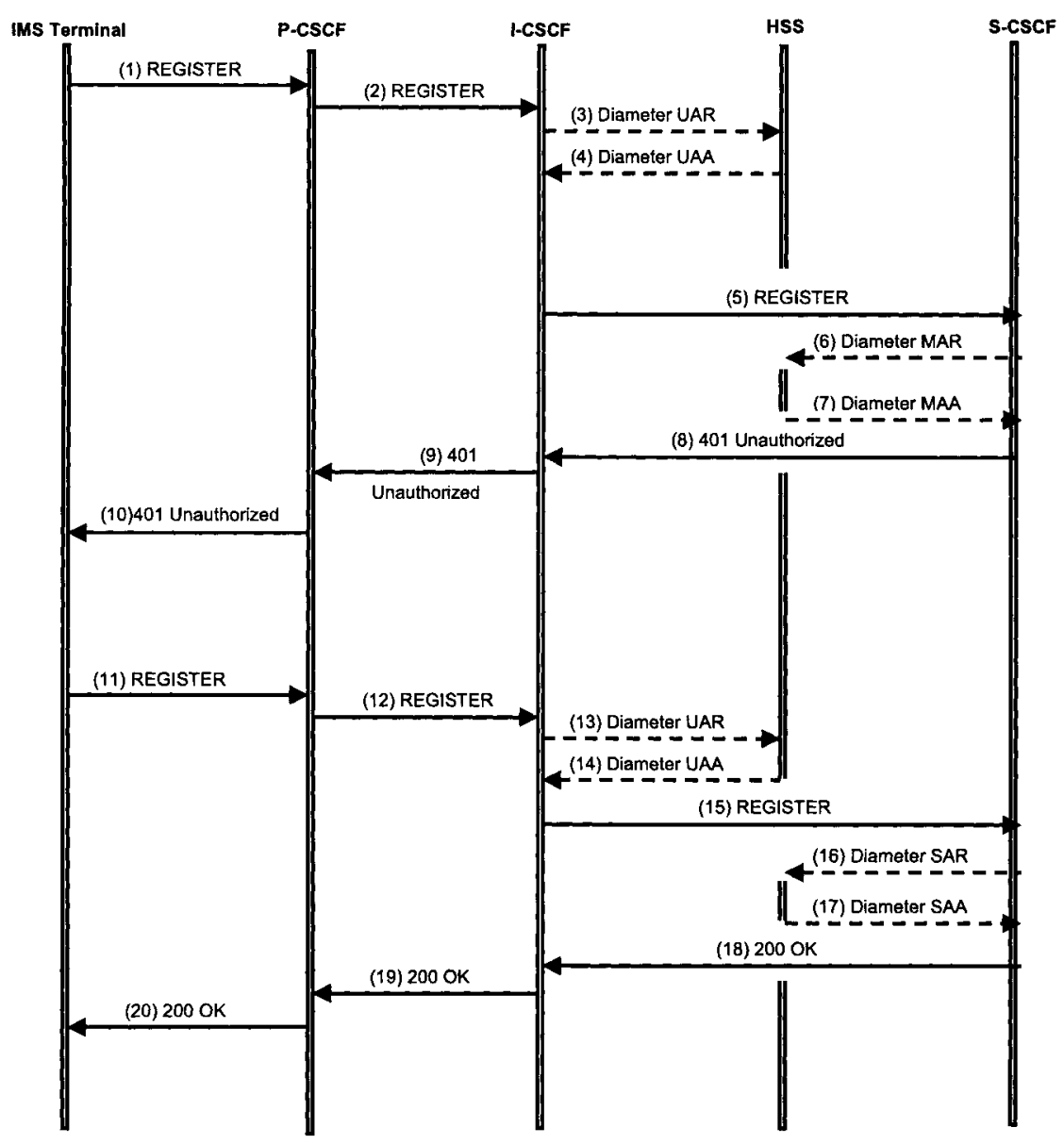

Fig. 5. IMS Authentication Process

The most important module in the IMS security is IP Multimedia Services Identity Module (ISIM), acts as storage for the secret key (K) and Authentication \& Key Agreement Algorithm (AKA). The ISIM is normally embedded on the Universal Integrated Circuit Card (UICC) like a smart card based device. The IMS security is based on a long-term secret key $(\mathrm{K})$ shared between the ISIM and the home Network Authentication Centre (AUC), and the AKA performs mutual authentication of ISIM and the AUC and generates Cipher Key (CK) and Integrity Key (IK). By this process the UE and home network have successfully authenticated and establishes a secure communication channel [4]. The device on which the ISIM resides is a temper-resistant and only physical access to it is not sufficient to result in exposing the secret key. It is further protected by the PIN code from unauthorized 
access. Thus the combination of ownership of physical device USIM/ISIM and knowledge of the secret pin code makes the security architecture of the IMS more robust [11]. The successful authentication and registration process is summarized in figure 5 .

\subsection{SA2: Security Management to Protect Gm-interface}

The Gm reference point connects the User to the IP Multimedia System Core. It is used to transport all Session Initiation Protocol (SIP) signaling messages between the $\mathrm{UE}$ and the P-CSCF. This security association manages secure link between the UE and a P-CSCF for the protection of the Gm reference point. The protection of this interface is very essential and therefore its security is considered very important. The IMS in 3GPP Releases 5 and 6 makes use of IPSec as the security mechanism between the P-CSCF and the UE. The Internet Protocol Security (IPSec) [12] is only one of several possible security mechanisms. The IMS was designed to allow alternative security mechanisms over the Gm interface as well. Allowing such openness usually creates backward compatibility problems because, for example, a Release 6-compliant UE would not be able to understand any alternative security mechanism, while it could be attached to a P-CSCF of a higher release that would already support alternatives to IPSec. Therefore, the SIP Security Mechanism Agreement (Sip-Sec-Agree) [13] was introduced to allow the UE and the P-CSCF to negotiate a common security mechanism for use between them. For current releases the only security mechanism is IPSec; however, it might be that some entities already support alternative mechanisms on a proprietary basis.

Authentication for IMS access is based on the AKA protocol but it cannot run directly over IP and requires a vehicle to carry protocol messages between the UE and the home network. SIP [14] acts as vehicle for AKA protocol and it is tunnelled inside SIP and therefore IMS access is obviously to authenticate it. During the authentication of the user, the UE and the IMS also negotiate the security mechanisms for securing subsequent SIP traffic in the Gm interface. SIP Security Mechanism Agreement (Sip-Sec-Agree) [13] is used for this security agreement and the UE and the P-CSCF exchange their respective lists of supported security mechanisms and the highest commonly supported one is selected. The selected security mechanism is used to provide data integrity protection.

Once the security mechanism has been selected and its use started, the previously exchanged list is replayed back to the network in a secure fashion. This helps the network to verify that the security mechanism selection was correct and the security agreement was not tampered with. An example of an attack that would be possible without this feature is bidding-down attack, where an attacker forces peers into selecting a known weak security mechanism [11]. The IPSec ESP (Encapsulated Security Payload) [15] provides both confidentially as well as data integrity and authentication which are mandatory in IP Multimedia System access security. AKA session keys are used as keys for the ESP SAs i.e. IK is used as the authentication key, and CK as the encryption key. 


\subsection{SA3: Security Management for $\mathrm{Cx}$ and Dx interfaces}

The Home Subscriber Server (HSS) stores subscriber and service data permanently and this centralized data is utilized by the I-CSCF and the S-CSCF when the user registers or receives sessions through $\mathrm{Cx}$ and $\mathrm{Dx}$ interfaces. SA3 provides security within the network domain internally for the Cx-interface between HSS \& S-CSCF, and for Dx-interface between HSS \& I-CSCF and the selected management protocol is Diameter [16] as shown in figure 6. The Diameter messages over the Cx and Dx interfaces make use of Stream Control Transmission Protocol (SCTP) [20] with IPSec for secure communication.

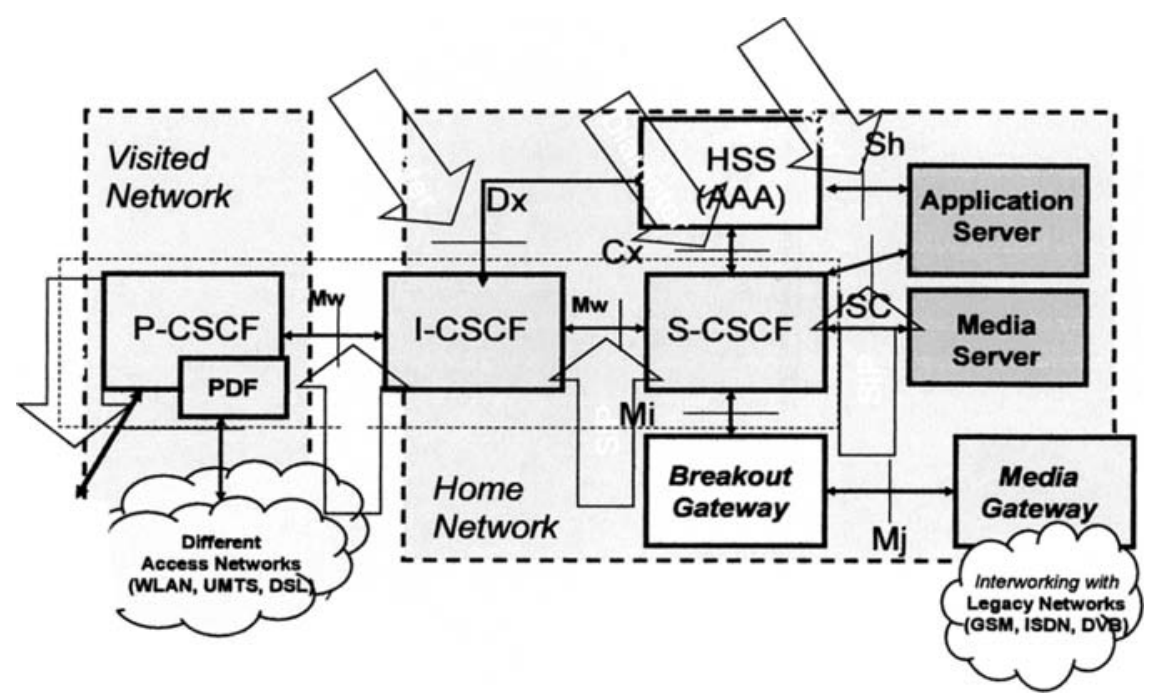

Fig. 6. SIP and Diameter Protocols Bindings

\subsection{SA4: Security Management for Roaming Users}

It provides security between P-CSCF and S-CSCF \& I-CSCF entities which are SIP capable nodes and only applicable when the P-CSCF resides in the visited network i.e. the user is roaming. The IP Multimedia System supports communication between home network and visited network, creating two scenarios weather the IMS terminal is in home network or roaming. In the first scenario the UE's first point of contact to the IMS, called P-CSCF is located in the home network and in the second scenario the $\mathrm{P}-\mathrm{CSCF}$ is located in the visited network (roaming). The visited network scenario is shown in figure 7. When P-CSCF resides in the visited network than by virtue of the AKA protocol, the shared secret is only accessible in the home network, which means that while authentication needs to take place in the visited network, certain delegation of responsibility needs to be assigned to the P-CSCF, as IPSec SAs exist between the P-CSCF and the UE. 


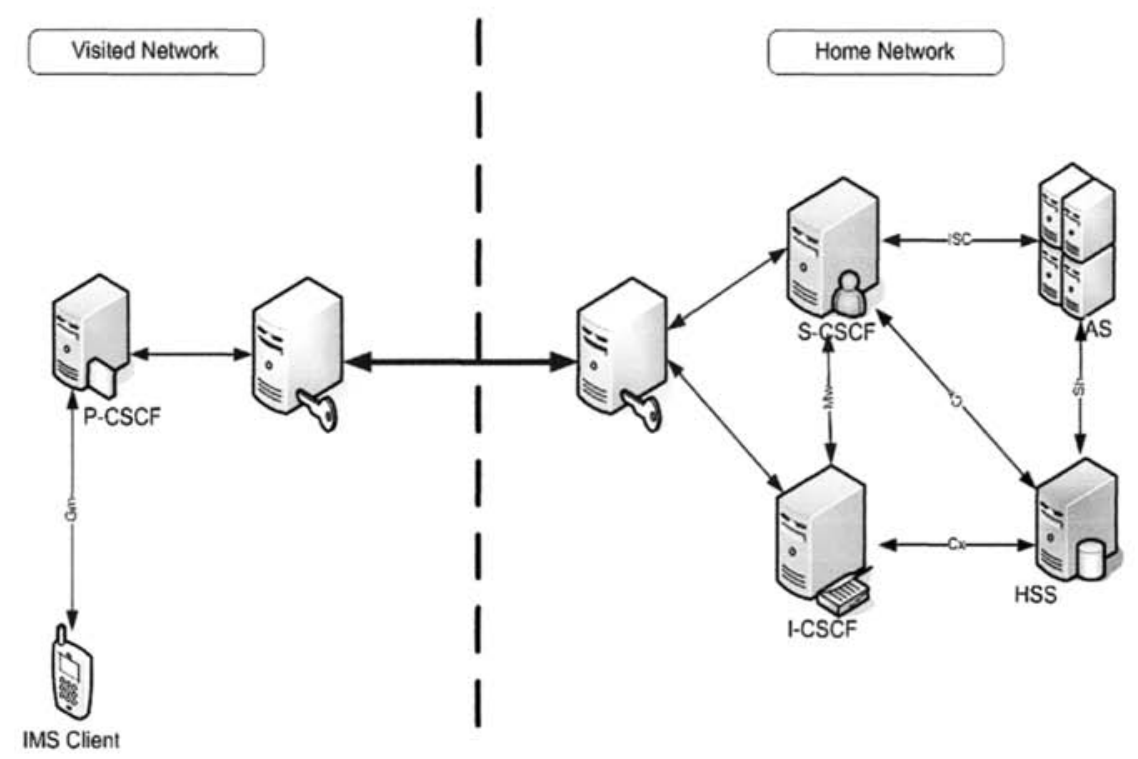

Fig. 7. IMS Roaming User

The traffic between the visited and home network are protected using the Network Domain Security/Internet Protocol (NDS/IP) at IP layer. The NDS/IP only protects traffic between network elements in the IP layer. The security gateway (SEG) enforces policy based security management on inbound and outbound traffic between the security domains like packet filtering or firewall functionality. All traffic within the IMS core network is routed via SEGs when the traffic is interdomain. For inter-domain IMS traffic protection, NDS/IP is mandatory to achieve confidentially and data integrity.

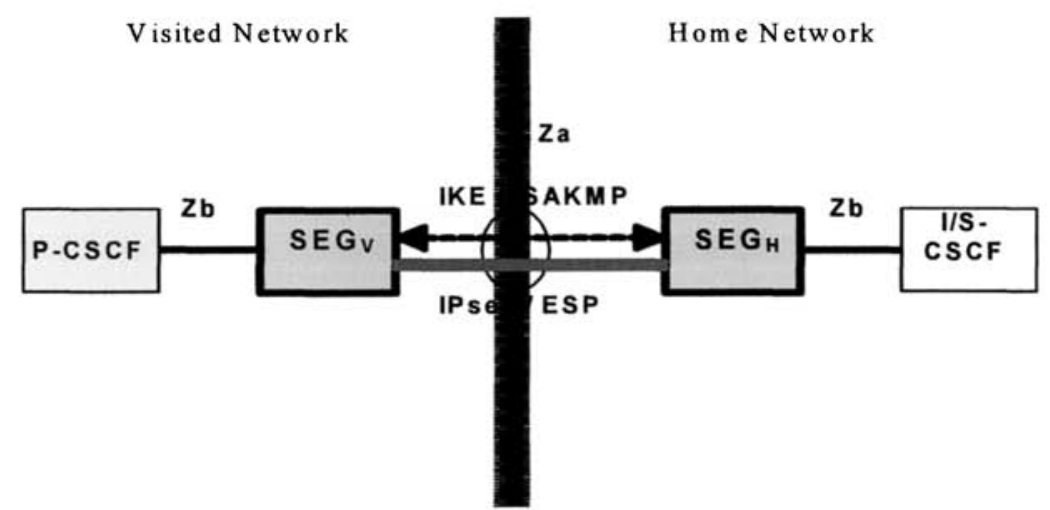

Fig. 8. Inter-Domains Security Gateways 
Each SEG is responsible for setting up and maintaining IPSec security associations [12] with its peer SEGs and performs negotiations using Internet Key Exchange (IKE) [17] protocol. Security gateway maintains two SAs per peer connection; one for inbound traffic and other for outbound traffic. The SEG also maintains a single Internet Security Association and Key Management Protocol (ISAKMP) SA [18], for key management and to build up the IPSec SAs between peer hosts as shown in figure 8 .

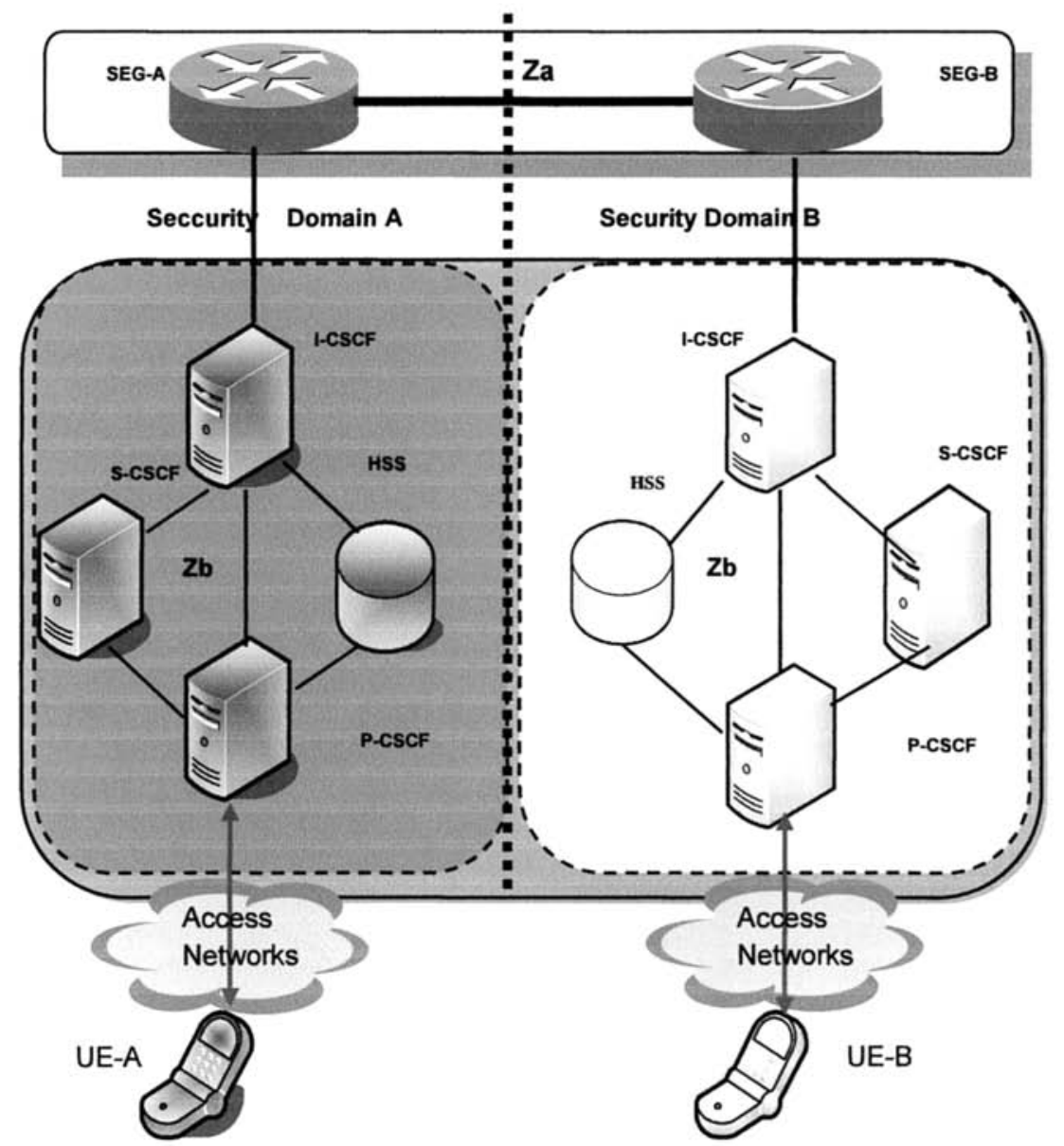

Fig.9. Concepts of Security Domains (Both Users are in their Home Domains) 


\subsection{SA5: Security Management for Home User}

This security association is among P-CSCF, S-CSCF and I-CSCF. It provides security management within the network internally between SIP capable nodes and only applicable when the P-CSCF resides in the home network. The IMS protects all IP traffic in the core network using Network Domain Security/IP (NDS/IP) which provides confidentially, data integrity, authentication and anti-replay protection for the traffic using combination of cryptographic security mechanisms and protocol security mechanisms applied in IP security (IPSec).

In the NDS/IP platform, the interfaces between elements inside security domain are denoted by $\mathrm{Zb}$ and the interfaces between different security domains are denoted by $\mathrm{Za}$ as shown in figure 9 . Use of the $\mathrm{Za}$ interface is always mandatory between different security domains while use of the $\mathrm{Zb}$ interface is optional and up to the security domain's administrator. Data authentication and integrity is mandatory for both interfaces, while use of encryption is recommended for the $\mathrm{Za}$ and optional for the $\mathrm{Zb}$ depending upon the nature of data contents.

\subsection{SA6: Security Management for Ut interface}

The Ut interface is the reference point between the User and the Application Server (AS) that enables users to securely manage and configure their network servicesrelated information hosted on an AS. Users can use the Ut reference point to create public service identities, such as a resource list, and manage the authorization policies that are used by the service. Examples of services that utilize the Ut reference point are presence and conferencing. The AS may need to provide security for the Ut reference point. HTTP is the chosen data protocol for the Ut reference point that performs the functionality to manage data traffic for HTTP based applications. Thus securing the Ut interface means to achieve confidentiality and data integrity protection of HTTP-based traffic.

The authentication and key agreement for the Ut interface is also based on AKA. The IMS defines the Generic Bootstrapping Architecture (GBA) [6] as a part of the Generic Authentication Architecture (GAA) that performs mutual authentication between Bootstrapping Server Functions (BSF) and the UE. AKA generates session keys and enable further applications provided by the Network Application Function (NAF). NAF issues subscriber certificates using an applications protocol secured by the bootstrapped session keys.

The authentication in the Ut interface is performed by authentication proxy. In terms of the GBA the authentication proxy is another type of NAF. Traffic in the Ut interface goes through the authentication proxy and is secured using the bootstrapped session key. The Ut interface employs the Transport Layer Security (TLS) [7] for both confidentiality and integrity protection. 


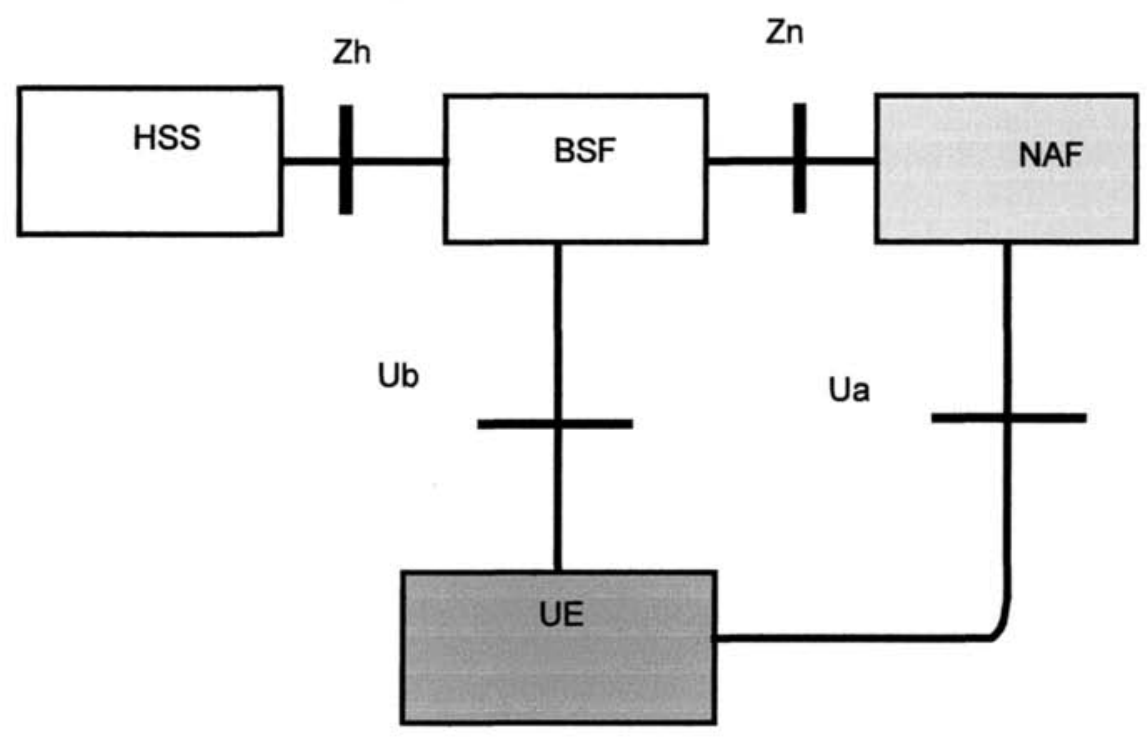

Fig. 10. Generic Bootstrapping Architecture

\subsection{SA7: Security Management for Access Networks}

This security association manages to protect the user and user's information on the access networks e.g. UMTS, GSM, GPRS, WLAN, DSL and VoIP. The security association takes place independently either in CS service domain or PS service domain. The network access security management architecture consists of User Service Identity Module (USIM), Mobile Equipment (ME), Access Network (AN), Service Network (SN) and Home Environment (HE) [19] as shown in figure 11. The USIM is required for accessing the Packet Switched (PS) domain in General Packet Radio System (GPRS) and identifies the particular subscriber. The USIM contains the security parameters for accessing the PS-domain, International Mobile Subscriber Identity (IMSI), list of allowed access points, MMS-related information.

In serving network, the Serving GPRS Support Node (SGSN) links the Radio Access Network (RAN) to the packet core network in the PS-service domain. It is responsible for performing both control and traffic handling functions for the PS domain. The control parts deal with mobility management and session management. The SGSN also ensures appropriate QoS and generates charging information. In the CS-service domain, the related part is Visitor Location Register (VLR). The authentication and key agreement procedure involves Authentication Centre (AUC) within HE, SGSN or VLR and Mobile Station (MS) networks entities [5]. 


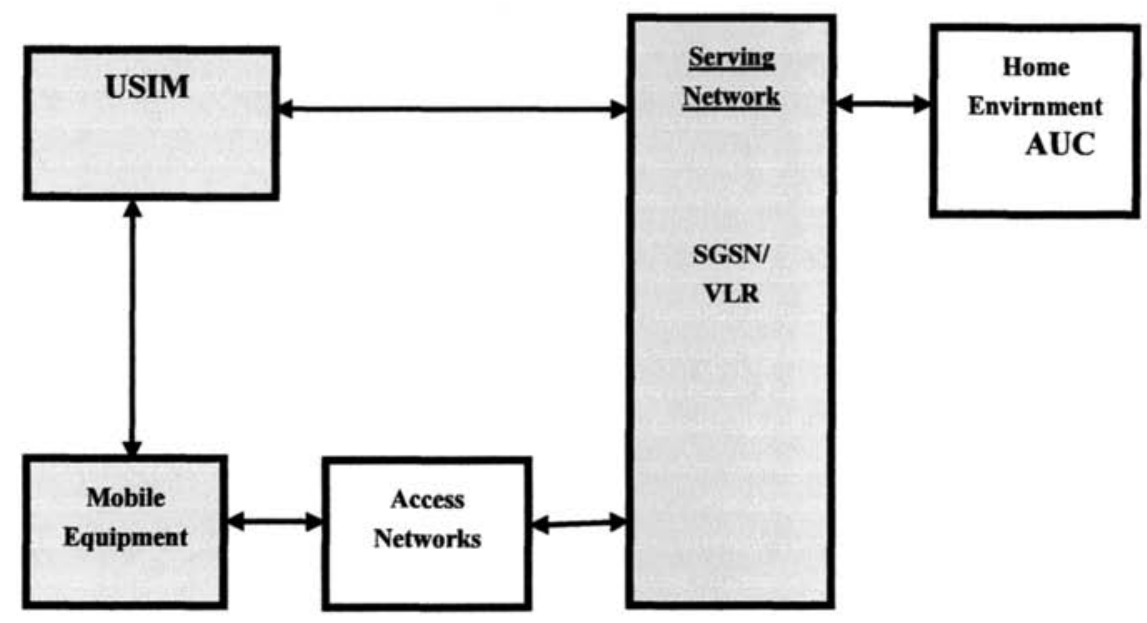

Fig. 11. Access Networks Architecture

\section{Conclusions}

This paper presents Security Associations Management (SAM) model which consists of seven security associations (AS1 to AS7) which are derived and based on different technical specifications of 3GPP and IETF to provide Secure Service Provisioning Framework (SSPF) for IP Multimedia System. The main focus is that we can be able to understand the complete security management for SIP based application as well as HTTP based services to establish a secure and protected environment based platform for IMS playground within $3 \mathrm{~Gb}\left(3^{\text {rd }}\right.$ Generation and beyond) Testbed at Fokus, Fraunhofer. The IMS Playground and $3 \mathrm{~Gb}$ Testbed provide research \& development platforms to scientists and engineers in the field of information technology, particularly for Next Generation Mobile Networks.

\section{References}

[1] About 3 Generation Partnership Project at http://www.3gpp.org

[2] M. Sher, T. Magedanz, "Secure Service Provisioning Framework (SSPF) for IP Multimedia System and Next Generation Mobile Networks" IWWST'05, $3^{\text {rd }}$ International Workshop in Wireless Security Technologies, London, U.K. April 2005, IWWST'05 Proceeding, ISSN 1746-904X, pp. 101-106. http://www.iwwst.org.uk.

[3] IMS Playground at www.fokus.fraunhofer.de/ims and $3 \mathrm{~Gb}$ Testbed at www.fokus.fraunhofer.de/national_host.

[4] 3GPP, TS 33.203 V6.4.0 (2004-09), 3G Security; "Access Security for IP-based Services (Release 6)". 
[5] 3GPP, TS 33.102 V6.2.0 (2004-09), 3G Security; "Security Architecture (Release 6)"

[6] 3GPP, TS 33.220 V6.2.0 (2004-09), "Generic Authentication Architecture (GAA); Generic Bootstrapping Architecture (Release 6)".

[7] 3GPP TS 33.222 V6.1.0 (2004-09), "Access to Network Application Functions using HTTP over TLS (HTTPS)".

[8] About 3 Generation Partnership Project 2 at http://www.3gpp2.org

[9] About TISPAN at http://portal.etsi.org/portal_common/home.asp?tbkeyl=TISPAN

[10] K. Knüttel, T.Magedanz, D. Witszek: "THE IMS PLAYGROUND @ FOKUS - AN OPEN TESTBED FOR NEXT GENERATION NETWORK MULTIMEDIA SERVICES", $1^{\text {st }}$ Int. IFIP Conference on Testbeds and Research Infrastructures for the DEvelopment of NeTworks and COMmunities (Tridentcom), Trento, Italien, Februar 23 - 25, 2005, Proceedings pp. 2 - I1, IBSN 0-7695-2219-x, IEEE Computer Society Press, Los Alamitos, California.

[11] Poikselkae, M., Mayer, G., Khartabil, H., Niemi, A., "The IMS, IP Multimedia Concepts and Services in the Mobile Domain", John Willey \& Sons Ltd, West Sussex, England, 2004.

[12] IETF RFC 2401, "IPSec Security Associations, SAs"

[13] IETF RFC 3329, "Security Mechanism Agreement for the Session Initiation Protocol (SIP)".

[14] IETF RFC 3261, "SIP: Session Initiation Protocol".

[15] IETF RFC 2406, "IPSec Encapsulating Security Payload, ESP".

[16] IETF RFC 3588, "Diameter Protocol".

[17] IETF RFC 2409, "IKE: Internet Key Exchange".

[18] IETF RFC 2408, "ISAKMP: Internet Security Associations and Key Management Protocol".

[19] M. Sher, T. Magedanz: "Network Access Security Management (NASM) Model for Next Generation Mobile Telecommunication Networks", IEEE/IFIP MATA'2005, $2^{\text {nd }}$ International Workshop on Mobility Aware Technologies and Applications - Service Delivery Platforms for Next Generation Networks, Montreal, Canada, October 17-19, 2005, Proceeding Springer-Verlag LNCS 3744-0263, ISSN: 0302-9743, Berlin Heidelberg 2005, pp. 263-272.

[20] IETF RFC 3554, "On the Use of Stream Control Transmission Protocol (SCTP) with IPSec". 